\title{
Multiobjective Shape Optimization with Constraints based on Estimation Distribution Algorithms and Correlated Information
}

\author{
Sergio Ivvan Valdez Peña \\ Center for Research in \\ Mathematics (CIMAT) \\ Department of Computer \\ Science \\ A.P. 402, Guanajuato, Gto. \\ 36000, México \\ ivvan@cimat.mx
}

\author{
Salvador Botello Rionda \\ Center for Research in \\ Mathematics (CIMAT) \\ Department of Computer \\ Science \\ A.P. 402, Guanajuato, Gto. \\ 36000, México \\ botello@cimat.mx
}

\author{
Arturo Hernández \\ Aguirre \\ Center for Research in \\ Mathematics (CIMAT) \\ Department of Computer \\ Science \\ A.P. 402, Guanajuato, Gto. \\ 36000 , México \\ artha@cimat.mx
}

\begin{abstract}
A new approach based on Estimation Distribution Algorithms for constrained multiobjective shape optimization is proposed in this article. Pareto dominance and feasibility rules are used to handle constraints. The algorithm uses feasible and infeasible individuals to estimate the probability distribution of evolving designs. Additionally, correlation among problem design variables is used to improve exploration. The design objectives are: minimum weight and minimum nodal displacement. Also, the resulting structures must fulfill three design constraints: a) maximum permissible Von Misses stress, b)connectedness of the structure elements, and c) small holes are not allowed in the structure. The finite element method is used to evaluate the objective functions and stress constraint.
\end{abstract}

\section{Categories and Subject Descriptors}

[TRACK: Estimation of Distribution Algorithms]: Area as CFP: EDA applications (interesting artificial and real-world problems)Area as CFP: design of hybrid methods by combining EDAs with other optimization methods

\section{General Terms}

Algorithm for Automatized Optimum Design

\section{Keywords}

Shape Optimization, MOEA, EDAs, Constraint Handling, Correlated Information

\section{INTRODUCTION}

Several authors have approached shape optimization problems $[2,4,3]$, principally by using genetic algorithms (GA). These approaches have proved their capacity to find approximate solutions. Two problems have prevented GA's from complete success: the lack of a "good" function to model all desirable features of the design; and poor exploration and premature convergence. In this work we present a method that improves search space exploration, therefore reducing premature convergence. Undesirable features such as small holes and unconnected elements are

Copyright is held by the author/owner.

GECCO'05, June 25-29, 2005, Washington, DC, USA.

ACM 1-59593-010-8/05/0006. prevented as well. These features are treated as problem constraints, which are handled by Pareto dominance criterion. Information from feasible and infeasible individuals is used to estimate the probability distribution of every representation bit $[7,1,9,8]$. Regularization is applied to the probability distributions (see [6]), in order to keep diversity (thus, reducing premature convergence and poor exploration).

\section{PROBLEM DEFINITION}

The multiobjective design goal is to find the set of structures with minimum weight and node displacements, which fulfill three design constraints: maximum Von Misses stress (standard mechanical design criterion, see [5]), small holes, and unconnected elements in the structure. Every solution is represented by a binary vector, each element in the binary vector represents one element, 0 is one element gap and 1 is presented element.

\section{OUTLINE OF THE ALGORITHM}

The Figure 1 shows the main procedures of the algorithm. First, we initialize the probability vectors $\hat{p}$. Then, every probability vector $p_{i}$ generates a subset of the population. Finally, all the non-dominated feasible individuals and some infeasible individuals are taken to update the probability distributions (the current Pareto set). When the probability distributions lost their exploration capacity, a procedure to regularize the probability vector must be done, using information about the neighborhood in every element. Then, when the probability regularization is done, the current Pareto set must be restarted with 0 individuals. Every generation the known Pareto set is updated to save the solutions (non-dominated and feasible individuals), obviously,it is updated finding the non-dominated individuals from the current Pareto set and the known Pareto set in every generation.

\section{RESULTS}

In Figure 3(a) is shown the load state of a experiment, some structures from any run are shown in Figure 3(b). 200 probability vectors, each one generating 10 individuals were used to find an average of 1580.83 vectors in the Pareto Set. The displacement was minimized at the 3 loaded nodes. In average, $3.928 \times 10^{6}$ function evaluations were computed. (The own weight is not considered (as a load) in the optimization problem).

\section{CONCLUSIONS}

A new approach for multiobjective shape optimization was presented, using Pareto dominance for constraint handling, the al- 


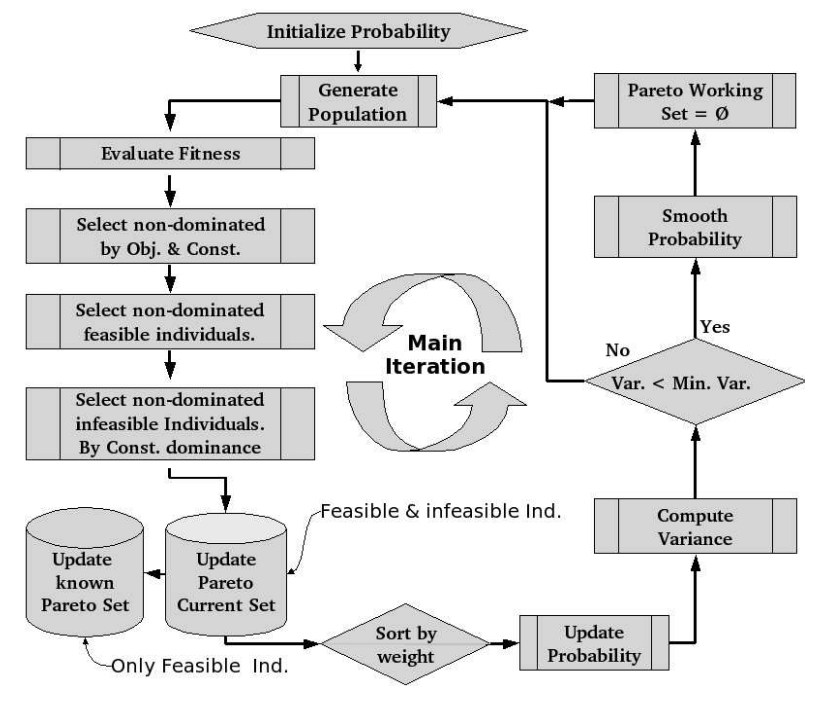

Figure 1: Main loop of algorithm depicting principal routines

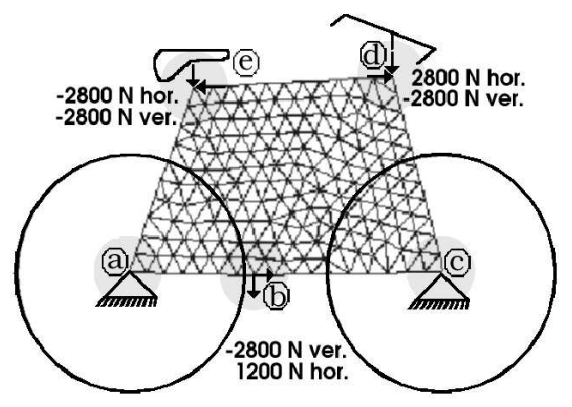

Figure 2: Load State of an experiment.

gorithm returns a set of solutions which fulfill the service conditions and minimize the structure weight and nodal displacement. Future work must explore how strong the optimization problem depends on the mesh, and how to initialize the probability distributions (after re-meshing the search space) in a multigrid strategy , considering mesh dependency and a search space reduction. The presented results show a good behavior and optimal solutions despite of the unstructured mesh (generally, other reported results use structured mesh). Finally, the future work considers the reduction of the computational cost by working with a fixed Pareto set size.

\section{Acknowledgements}

The authors acknowledge support for this work from National Council of Science and Technology of México, CONACyT, Project Number 40721-Y

\section{REFERENCES}

[1] S. Baluja. Population based incremental learning: A method for integrating genetic search based function optimization and competitive learning. School of Computer Science Carnegie Mellon University, Pittsburgh, Pennsylvania 1523, CMU-CS-94-163, 1996.

[2] C. Chapman, K. Saitou, and M. Jakiela. Genetic algorithms as an approach to configuration and topology design. Journal of Mechanical Design, 116:1005-11, 1994.

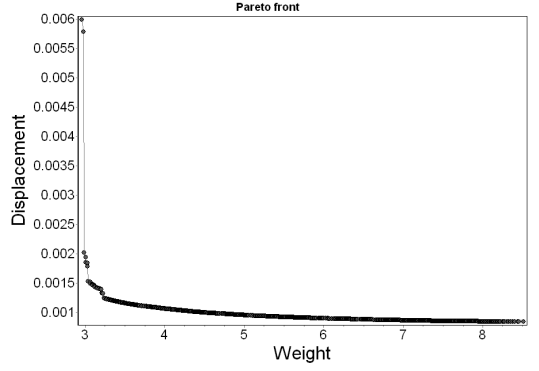

(a)

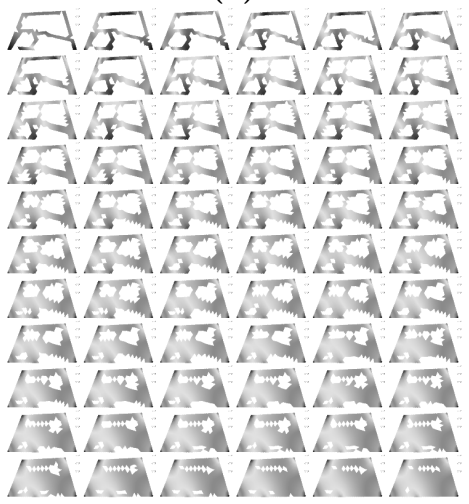

(b)

Figure 3: (a) Pareto front from a typical run, (b) Some structures on the Pareto front from a typical run

[3] K. Deb and T. Goell. Multiobjetive evolutionary algorithms for engineering shape optimization. KanGal report, (200003), 2000.

[4] C. Kane and M. Schoenauer. Topological optimum design using genetic algorithms. Control and Cybernetics, 25(5), 1996.

[5] L. Malvern. Introduction to the Mechanics of a Continuous Medium. Pretence Hall Inc., Englewood Cliffs New Jersey, 1969.

[6] J. Marroquín, M. Rivera, and M. Nakamura. Gauss-Markov measure field models for low-level vision. IEEE Transactions On PAMI, 23(4):337-348, 2001.

[7] H. Muhlenbein and G. PaaB. From recombination of genes to the estimation of distributions with binary parameters. Parallel problem Solving form Nature, PPSN(IV):178-187, 1996.

[8] M. Pelikan, D. Goldberg, and C. Paz. Linkage problem, distribution estimation and Bayesian networks. IlliGal Report, (98013), 1998.

[9] M. Pelikan, D. Goldberg, and C. Paz. BOA: The Bayesian optimization algorithm. In Proceedings of the Genetic and Evolutionary Computation Conference, 1999. 